\title{
Approximate Shortest Path Queries Using Voronoi Duals*
}

\author{
Shinichi Honiden Michael E. Houle Christian Sommer ${ }^{\dagger} \quad$ Martin Wolff
}

November 13, 2009

\begin{abstract}
We propose an approximation method to answer point-to-point shortest path queries in undirected edge-weighted graphs, based on random sampling and Voronoi duals. We compute a simplification of the graph by selecting nodes independently at random with probability $p$. Edges are generated as the Voronoi dual of the original graph, using the selected nodes as Voronoi sites. This overlay graph allows for fast computation of approximate shortest paths for general, undirected graphs. The time-quality tradeoff decision can be made at query time. We provide bounds on the approximation ratio of the path lengths as well as experimental results. The theoretical worst-case approximation ratio is bounded by a logarithmic factor. Experiments show that our approximation method based on Voronoi duals has extremely fast preprocessing time and efficiently computes reasonably short paths.
\end{abstract}

\section{Introduction}

We wish to answer shortest path queries for large edge-weighted graphs such as those stemming from transportation networks, social networks, protein interaction networks, and the web graph. One could use a classical single source shortest path algorithm such as Dijkstra's [Dij59], which has worst-case running time $O(m+n \lg n)$, where $n$ denotes the number of nodes and $m$ the number of edges. However, for large graphs, only a relatively small portion of the graph can be searched at query time. If preprocessing is allowed, queries can be answered much more quickly. The algorithms with fastest query times are those that precompute and store the shortest-path distances between all possible pairs of source and target - that is, those that precompute solutions to the All Pairs Shortest Path Problem. Shortest path queries could then be answered in constant time. The fastest known algorithm for computing all shortest paths runs in time $O\left(n^{3} / \lg ^{2} n\right)$ [Cha07]. Unfortunately, the preprocessing time is prohibitively large in practice.

The goal is to mediate between the two extremes of no precomputation of paths and total precomputation of paths. The desired tradeoff between preprocessing time and query time depends on the needs of the application.

\section{$1.1 \quad$ Related work}

In the following, we give a brief overview of related work for shortest path and distance queries.

\footnotetext{
*An extended abstract appeared in the proceedings of the 6th annual International Symposium on Voronoi Diagrams in Science and Engineering (ISVD 2009)

${ }^{\dagger}$ Correspondence to sommer@nii.ac.jp.
} 
Theoretical. Data structures allowing for shortest path or distance queries are referred to as distance oracles. Their construction is closely related to that of graph spanners. For a pair of nodes $(s, t)$, an approximate distance oracle is said to have stretch $(\alpha, \beta)$ if it returns a distance in the range $[d(s, t), \alpha \cdot d(s, t)+\beta]$ [EP04]. A girth conjecture by Erdős implies that, for general undirected graphs, distance oracles with multiplicative stretch $\alpha<2 k+1$ need $\Omega\left(n^{1+1 / k}\right)$ space. An algorithm by Thorup and Zwick [TZ05] constructs such an oracle in expected time $O\left(k m n^{1 / k}\right)$ with query time $O(k)$ and stretch $(2 k-1,0)$. For constant $k$, except for the preprocessing time, all their bounds are essentially tight. Baswana and Kavitha [BK06] provide a solution with preprocessing time of $O\left(n^{2} \lg n\right)$. For unweighted graphs, subquadratic preprocessing time is possible [BGSU08]. For planar (directed) graphs with integer weights, an algorithm by Thorup [Tho04a] constructs a $(1+\epsilon, 0)$-stretch oracle in time $O\left(n \lg ^{3} n \lg (n \Delta)\right)$, where $\Delta$ denotes the largest weight. Unfortunately, for huge non-planar graphs, these results are not practical.

Practical. The main focus of practical investigations so far has been on large road networks. There has been considerable recent progress: for the road networks of Europe or the USA, using a high-performance computer, a speed-up of several orders of magnitude compared to Dijkstra's algorithm can be achieved with a preprocessing time in the tens of minutes [SS07a]. Unfortunately, theoretical bounds on both query time and preprocessing time are often difficult to obtain. Goldberg and Harrelson [GH05] proposed a variant of $\mathrm{A}^{*}$ search [Dor67] in which distances are precomputed with respect to a small set of 'landmark' vertices. Hierarchical methods [GSSD08, SS07b] provide an efficient framework, especially in the case of road networks. Sanders and Schultes [SS07a, SS07b, SS06] developed a method to compute shortest paths in 'almost constant time' with a carefully designed structure consisting of precomputed shortest paths. Their solution is tailored to perform exceptionally well for road networks, where graphs are almost planar and nodes have small constant degrees. Precomputation is time- and space-consuming; however, it is still manageable in practice, and allows for extremely fast query times.

Even though road networks constitute the most common and popular application of shortest path query algorithms to date, other challenging applications exist. Computer networks, social networks, protein interaction networks, and the web graph exhibit different degree and structural properties, and may contain hundreds of millions or even billions of nodes. In specific cases, a user might be willing to trade preprocessing time against exactness due to the vast size of the data or due to restricted processing power. These scenarios may require the use of a fast approximation method.

\subsection{Contribution}

We propose an approximation method to answer shortest path queries in general, undirected graphs with positive edge weights, based on random sampling and graph Voronoi duals [Meh88, Erw00]. In preprocessing, each node is selected as a Voronoi site independently at random with probability $p$, and the Voronoi dual is computed for the selected sites (Section 3). This preprocessing step is very efficient; it takes time proportional to computing one single source shortest path tree (Section 4). For $p<1$, the resulting dual graph is expected to be smaller than the original graph. At query time, search for the shortest path from source $s$ to target $t$ can potentially be done faster in the Voronoi dual. We let the shortest path in the Voronoi dual guide the search for an approximate shortest path in the original graph. We prove that the expected approximation ratio is at most logarithmic in the number of nodes on the actual shortest path, and that this bound is tight (Section 5). Our experimental results show that, in practice, the approximation is much better than the stated 
theoretical bound and that the preprocessing overhead is indeed extremely low (Section 6).

\section{Preliminaries}

An edge-weighted graph $G=(V, E, \omega)$ consists of a graph $(V, E)$ together with a weight function $\omega: E \rightarrow \mathbb{R}$. We assume positive edge weights; that is, $\omega: E \rightarrow \mathbb{R}^{+}$. For the remainder of the paper, we will refer to the number of nodes and edges of the graph by $n=|V|$ and $m=|E|$, respectively.

A path from $s=u_{0} \in V$ to $t=u_{h} \in V$ is a node sequence $\left(u_{0}, u_{1}, \ldots, u_{h}\right)$ for which $\left(u_{i}, u_{i+1}\right) \in$ $E$ for all $i \in\{0,1, \ldots h-1\}$. The length of a path $P$ is the sum of its edge weights $\ell(P):=$ $\sum_{i=0}^{h-1} \omega\left(u_{i}, u_{i+1}\right)$. A subpath $P^{\prime}$ of a path $P$ is a subsequence of its nodes $P^{\prime}=\left(u_{i}, u_{i+1}, \ldots u_{j}\right)$, $0 \leq i<j \leq h$. A simple path is a path without repeated vertices. Let $\mathcal{P}_{G}(u, v)$ denote the set of paths from $u$ to $v$ in $G$. The distance $d(u, v)$ between two nodes $u, v$ is the length of a shortest path from $u$ to $v$; that is, $d(u, v)=\min _{P \in \mathcal{P}(u, v)} \ell(P)$. If $\mathcal{P}(u, v)=\emptyset$ then $d(u, v):=\infty$. Let $S P_{G}(s, t)$ be an arbitrary shortest path from $s$ to $t$. Analogously to the multiplicative stretch of a distance oracle, we define the stretch of a path $P$ from $s$ to $t \neq s$ as the ratio $\ell(P) / \ell\left(S P_{G}(s, t)\right)$.

\subsection{Graph Voronoi Diagram}

The classical Voronoi diagram is a distance-based decomposition of a metric space relative to a discrete set, the Voronoi sites [Dir50, Vor07]. For a survey on this fundamental structure, we refer to [Aur91]. Among many applications, the Voronoi diagram is often used to solve facility location problems [Sha75, ACS99, AGK ${ }^{+}$04, GKP05, Svi08]. The Voronoi diagram and the Delaunay triangulation of $n$ points in the plane can be computed in expected time $n \cdot 2^{O(\sqrt{\lg \lg n})}$ [CP07], which is even faster than $O(n \lg n)$.

Mehlhorn [Meh88] and Erwig [Erw00] proposed an analogous decomposition, the Graph Voronoi Diagram, for undirected and directed graphs respectively. Since the Voronoi diagram for the Euclidean space is used for various applications, its graph counterpart, the graph Voronoi diagram, may be used for these applications if the underlying metric is the shortest path metric of a graph. Real-world distances or travelling times can be approximated more appropriately using models based on weighted graphs. In general, non-planar networks such as social networks, computer networks, protein interaction networks, and the web graph cannot be embedded into a low-dimensional Euclidean space without significant distortion.

Definition 1 (Graph Voronoi Diagram [Meh88, Erw00]). In a graph $G=(V, E, \omega)$, the Voronoi diagram for a set of nodes $K=\left\{v_{1}, \ldots, v_{k}\right\} \subseteq V$ is a disjoint partition $\operatorname{Vor}_{(G, K)}:=\left\{V_{1}, \ldots, V_{k}\right\}$ of $V$ such that for each node $u \in V_{i}, d\left(u, v_{i}\right) \leq d\left(u, v_{j}\right)$ for all $j \in\{1, \ldots, k\}$.

The $V_{i}$ are called Voronoi regions. The graph Voronoi diagram is not necessarily unique, as a node $u$ may have the same distance to more than one Voronoi node. Let vor $(u)$ denote the index $i$ of the Voronoi region $V_{i}$ containing $u$; that is, $\operatorname{vor}(u)=i \Leftrightarrow u \in V_{i}$.

Analogously to the Delaunay triangulation dual for classical Voronoi diagrams of point sets, we define the Voronoi dual for graphs.

Definition 2. Let $G=(V, E, \omega)$ be an edge-weighted graph and $\operatorname{Vor}_{G, K}$ its Voronoi diagram. The Voronoi dual is the graph $G^{*}=\left(K, E^{*}, \omega^{*}\right)$ with edge set $E^{*}:=\left\{\left(v_{i}, v_{j}\right): v_{i}, v_{j} \in K\right.$ and $\exists u \in$ $\left.V_{i} \wedge \exists w \in V_{j}:(u, w) \in E\right\}$, and edge weights $\omega^{*}\left(v_{i}, v_{j}\right):=\min _{\substack{u \in V_{i}, w \in V_{j} \\(u, w) \in E}}\left\{d\left(v_{i}, u\right)+\omega(u, w)+d\left(w, v_{j}\right)\right\}$. 
Figure 1 illustrates two graph Voronoi diagrams for the same (planar) graph but with different edge weights. Although the classical Voronoi dual of a non-degenerate set of points in the plane is always a triangulation, the graph Voronoi dual is not necessarily a triangulation, even for planar graphs. For example, a graph Voronoi dual may have nodes whose removal would disconnect the graph.
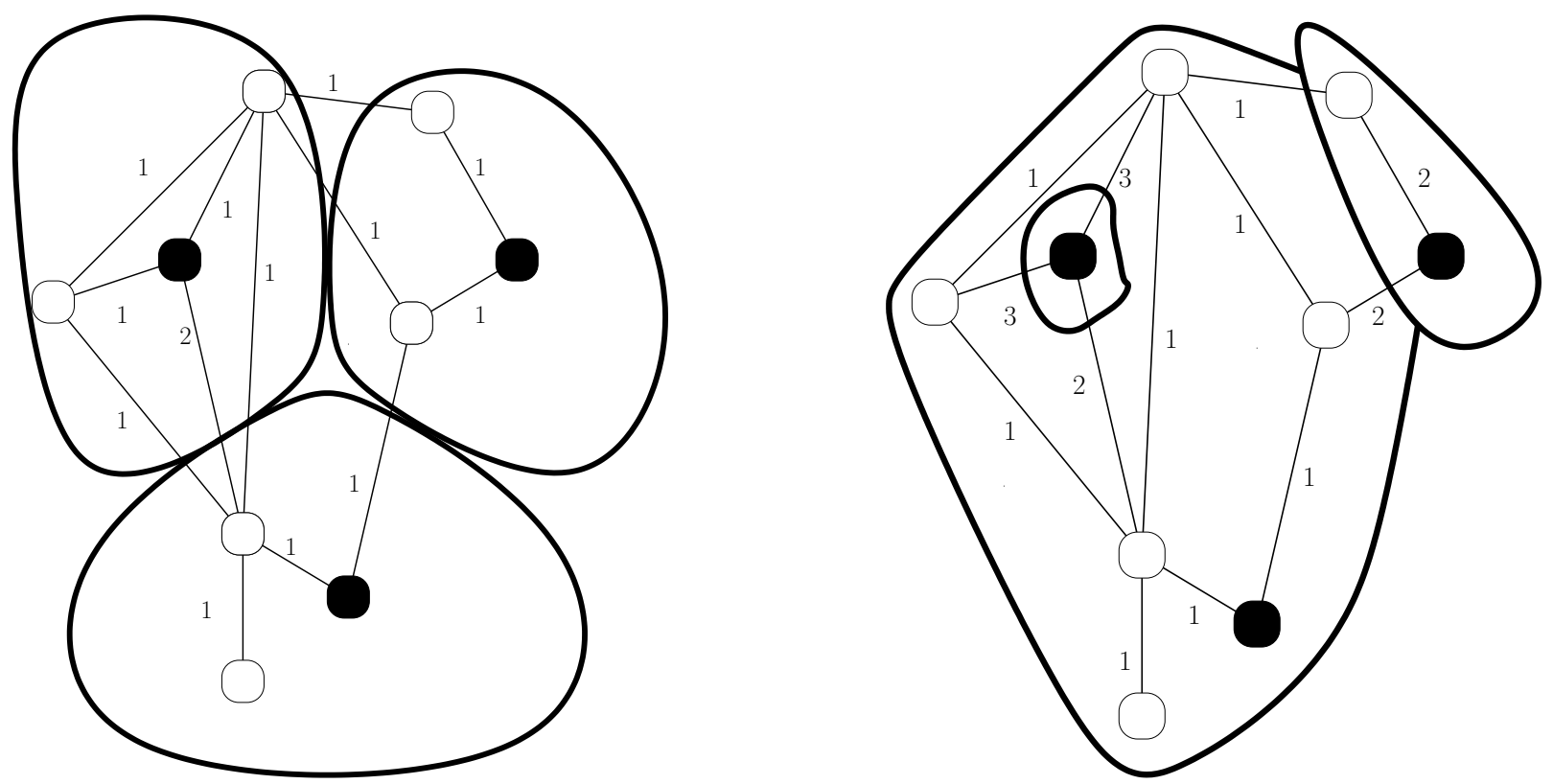

Figure 1: Two graph Voronoi diagrams for the same planar graph but with different edge weights. Voronoi nodes are black and the remaining nodes are white. Even though the graphs are structurally equivalent, the corresponding graph Voronoi diagrams are not.

Erwig [Erw00, Theorem 2] showed that the graph Voronoi diagram can be constructed with a single Dijkstra search in time $O(m+n \cdot \lg n)$. A heap is used to store the shortest path distances from nodes to their closest Voronoi node. The heap is initialized to store the Voronoi nodes themselves. Thereafter, as long as there are nodes in the queue, the minimum is extracted from the heap and processed (or 'settled') by assigning to it a Voronoi region, storing the distance to its Voronoi node, and adding to or updating its neighbors in the queue. We slightly modify this construction of the Voronoi diagram [Erw00, Section 3.1] to compute the Voronoi dual - that is, to also compute $E^{*}$ and $\omega^{*}$. Whenever a node $u$ is settled in the Dijkstra search, for all its settled neighbors $u^{\prime}$ of different Voronoi regions, the edge $\left(v_{\operatorname{vor}(u)}^{*}, v_{\operatorname{vor}\left(u^{\prime}\right)}^{*}\right)$ with weight $\omega_{G^{*}}\left(v_{\operatorname{vor}(u)}^{*}, v_{\operatorname{vor}\left(u^{\prime}\right)}^{*}\right)=$ $d_{G}\left(v_{\operatorname{vor}(u)}, u\right)+\omega_{G}\left(u, u^{\prime}\right)+d_{G}\left(u^{\prime}, v_{\operatorname{vor}\left(u^{\prime}\right)}\right)$ is added, or its length is decreased if there already is an edge in $G^{*}$ representing a longer path in $G$. This modification of Erwig's algorithm is shown as Algorithm 1.

In the analysis to follow (in Section 5) we move back and forth between a graph and its dual. For this we need the following definitions.

Definition 3. Given a path $P=\left(u_{0}, u_{1}, \ldots, u_{h}\right)$, the Voronoi path of $P$ is the sequence of vertices $P^{*}=\left(v_{\operatorname{vor}\left(u_{0}\right)}, v_{\operatorname{vor}\left(u_{1}\right)}, \ldots, v_{\operatorname{vor}\left(u_{h}\right)}\right)$. 


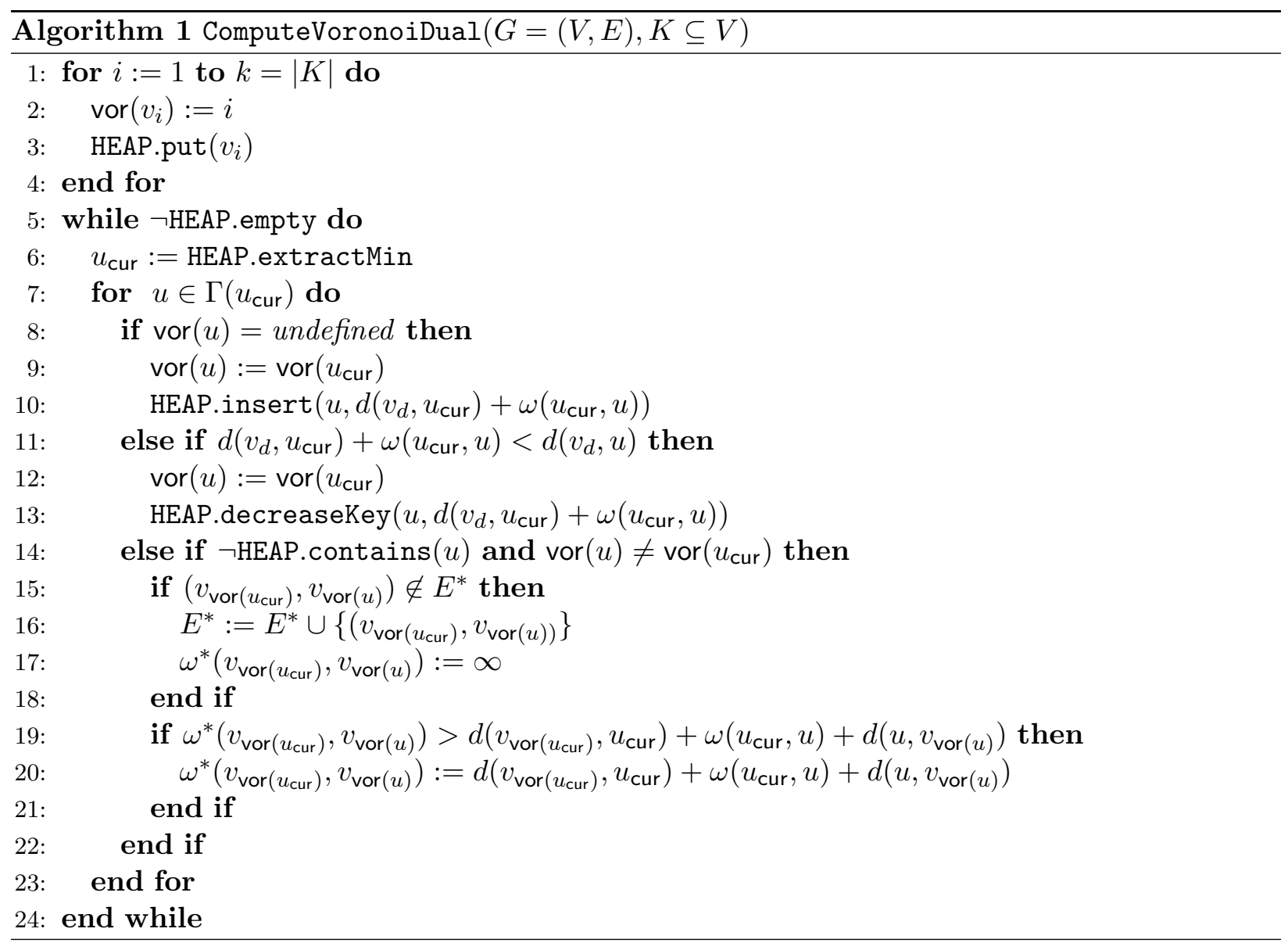

Note that the Voronoi path $P^{*}$ may not necessarily be simple, as multiple consecutive occurrences of nodes $v_{\operatorname{vor}\left(u_{i}\right)}$ are possible in $P^{*}$. They are treated as a single occurrence, and such paths are deemed to be equivalent.

Lemma 1. For any path $P=\left(u_{0}, \ldots, u_{h}\right)$ in an undirected graph $G=(V, E, \omega)$, the corresponding Voronoi path $P^{*}$ exists and is unique.

Proof. Suppose that there is no such path $P^{*}$ in $G^{*}$. This implies that there exist pairs of nodes $u_{i}, u_{i+1}$ on the path $P$ for which $v_{\operatorname{vor}\left(u_{i}\right)} \neq v_{\operatorname{vor}\left(u_{i+1}\right)}$ and $\left(v_{\operatorname{vor}\left(u_{i}\right)}, v_{\operatorname{vor}\left(u_{i+1}\right)}\right) \notin E^{*}$. As $u_{i}, u_{i+1}$ are consecutive nodes on the path $P$, we know that $\left(u_{i}, u_{i+1}\right) \in E$. This contradicts the definition of the Voronoi dual (Def. 2), since $\left(u_{i}, u_{i+1}\right) \in E$ and $v_{\text {vor }\left(u_{i}\right)} \neq v_{\text {vor }\left(u_{i+1}\right)}$ together imply that $\left(v_{\operatorname{vor}\left(u_{i}\right)}, v_{\operatorname{vor}\left(u_{i+1}\right)}\right) \in E^{*} . \quad P^{*}$ is unique since each node $u_{i}$ on the path belongs to exactly one Voronoi region, corresponding to exactly one Voronoi node $v_{\operatorname{vor}\left(u_{i}\right)}$.

Definition 4. For a path $P^{*}$ in the Voronoi dual $G^{*}$ of a graph $G$, the Voronoi sleeve is the subgraph of $G$ induced by the nodes in the union of all Voronoi regions $V_{i}$ for which its Voronoi node $v_{i}$ lies on $P^{*}$,

$$
\text { Sleeve }_{\left(G, G^{*}\right)}\left(P^{*}\right):=G\left[\bigcup_{v_{i} \in P^{*}} V_{i}\right] .
$$


With the definitions at hand we can now state the approximation method.

\section{The Method}

In preprocessing, each node is selected as a Voronoi site independently at random with probability $p$, and the Voronoi dual is computed for the selected sites (Algorithm 2). For the sake of exposition, we treat the computation of the Voronoi dual as a 'black box', denoted by ComputeVoronoiDual.

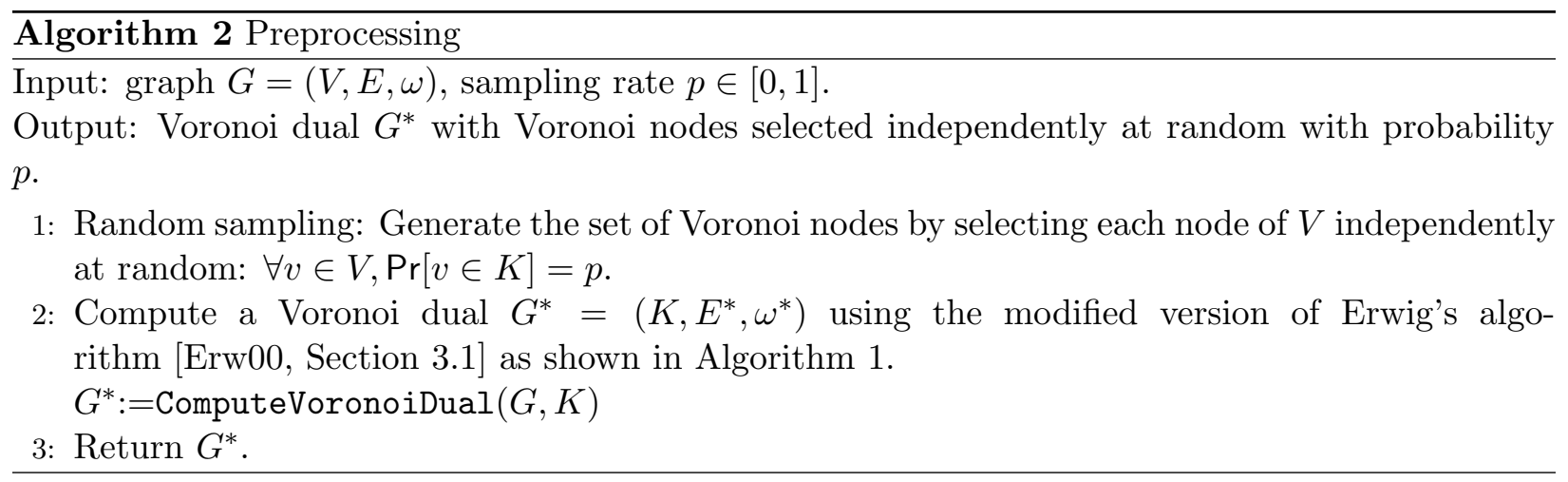

Lemma 2. For a graph $G=(V, E)$ with $n:=|V|$ and $m:=|E|$, Algorithm 2 takes time proportional to that of Dijkstra's single source shortest path algorithm.

Proof. Erwig's variant of Dijkstra's algorithm computes the graph Voronoi diagram in a worstcase time proportional to Dijkstra's algorithm [Erw00, Theorem 2]. The only modification of Algorithm 1 compared to Erwig's variant is the following: for each node, at the time it is settled, all its neighbors are inspected. Therefore, each edge is additionally considered two times in total. This yields the same asymptotic running time.

The preprocessing time complexity is proportional to the cost of computing one single source shortest path tree. Details are discussed in Section 4.

At query time, given a graph $G$ and its Voronoi dual $G^{*}$ we answer (approximate) shortest path queries between source $s$ and target $t$, by first searching for a shortest path $S P_{G^{*}}\left(v_{\operatorname{vor}(s)}, v_{\operatorname{vor}(t)}\right)$ in the smaller Voronoi dual $G^{*}$. This path determines the subgraph $\mathcal{S}=\operatorname{Sleeve}\left(S P_{G^{*}}\left(v_{\operatorname{vor}(s)}, v_{\operatorname{vor}(t)}\right)\right)$, whose shortest path $S P_{\mathcal{S}}(s, t)$ approximates the shortest path $S P_{G}(s, t)$ in $G$. The shortest path in the Voronoi dual guides the Dijkstra search in the original graph. For a pseudo-code description, see Algorithm 3.

The running time of Algorithm 3 depends on $G$ and $p$. Let $N^{*}$ and $M^{*}$ denote the random variables measuring the number of nodes and edges of the Voronoi dual. Clearly $E\left[N^{*}\right]=p \cdot n$. The expected query time without refinement (computing the shortest path in the Voronoi sleeve) is at most $O\left(N^{*} \lg N^{*}+M^{*}\right)$. The time for the refinement step depends on the size of the Voronoi sleeve. The analysis will show that the refinement step is not necessary for the approximation ratio to hold for long distance queries; however, it makes a practical difference for the quality of paths. For $p=O\left(n^{-2 / 3}\right), E\left[N^{*}\right]=O\left(n^{1 / 3}\right)$, and thus we can afford to compute all-pairs shortest path distances in the Voronoi dual $G^{*}$ in overall linear expected time. This allows for constant-time approximate distance queries. 


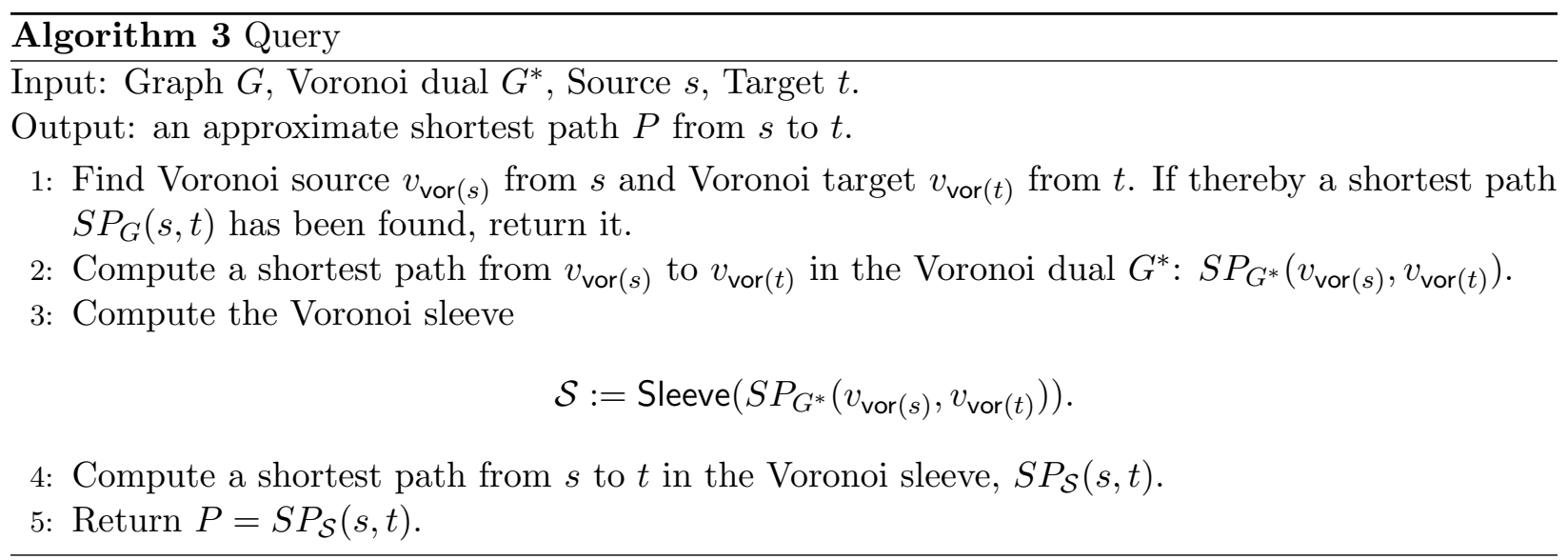

\begin{tabular}{|c|c|}
\hline Time & Reference \\
\hline$O(m \lg n)$ & [Wil64] \\
\hline$O(m+n \lg n)$ & [FT87] \\
\hline$O(m \sqrt{\lg n})$ & [FW93] \\
\hline$O\left(m+n \frac{\lg n}{\lg \lg n}\right)$ & [FW93] \\
\hline$O(m \lg \lg n)$ & [Tho00b] \\
\hline$O\left(m+n \lg ^{1 / 2+\epsilon} n\right)$ & [Tho00b] \\
\hline$O(m+n \sqrt{\lg n \lg \lg n})$ & [Ram96] \\
\hline$O\left(m+n \lg ^{1 / 3+\epsilon} n\right)$ & [Ram97] \\
\hline$O(m+n \lg \lg n)$ & [Tho04b] \\
\hline
\end{tabular}

Table 1: Running times for different implementations of Dijkstra's algorithm, excerpted from [Tho99, p. 364]. The algorithms in the first two rows work for both the pointer machine and the RAM model. The analysis of the algorithms from row 3 onwards only works in the RAM model.

\section{Computational Complexity}

In this section we study the cost of computing a Voronoi dual. Recall that in Erwig's algorithm [Erw00, Section 3.1] the graph Voronoi diagram is constructed with a single Dijkstra search. A heap is used to store the shortest path distances from nodes to their closest Voronoi node. Conceptually, a dummy node with a zero-weighted edge to each of the Voronoi nodes is added, the dummy node is inserted into the heap, and the Dijkstra single source shortest path search is executed. The running times of different implementations of Dijkstra's algorithm depend on the priority queue employed (see Table 1). Using Fibonacci heaps [FT87], Dijkstra's algorithm takes time $O(m+n \lg n)$.

Erwig also claims a time lower bound of $\Omega(\max (n,(n-k) \lg k))$ [Erw00, Theorem 1]. The lower bound simplifies to $\Omega(n \lg n)$ when the number of Voronoi nodes is assumed to be $k=n^{C}$ for a fixed choice of $C \in(0,1)$. Assuming that all edges must be inspected at construction time, this lower bound would be tight. The bound is information theoretic: for a connected graph, each node $w \in V \backslash K$ is in exactly one of the $k$ regions $V_{i}$. Encoding one instance out of these $k^{n-k}$ possibilities requires $\lg k^{n-k}=(n-k) \lg k$ bits. 
For some graphs with special properties, Erwig's lower bound may not apply. Eppstein and Goodrich [EG08] presented a linear-time algorithm to compute the Voronoi diagram for road networks satisfying certain geometric properties. Also, the lower bound may not hold under different models of computation, such as the word RAM model. This model assumes that basic operations such as adding two words requires a single time step, and that the time compexity is the number of word operations executed. The space complexity is the number of words of storage required, assuming that any identifier (such as a node label) or value (such as a distance) can be contained in a single word. Under the word RAM model, the implementation of Dijkstra's algorithm by Thorup [Tho04b] requires only $O(m+n \lg \lg n)$-time.

Corollary 1. The graph Voronoi dual can be computed in time $O(m+n \lg \lg n)$ in the word $R A M$ model.

Note that the time upper bound under the word RAM model does not contradict Erwig's information-theoretic lower bound [Erw00, Theorem 1] of $\Omega(n \lg n)$ bits.

Computing a graph Voronoi dual does not actually require the use of Dijkstra's algorithm any single source shortest path algorithm (including parallel and distributed algorithms) can be used to compute a graph Voronoi dual as follows. Instead of an adapted Dijkstra search, we may also

1. augment $G$ by introducing a dummy node $v_{d}$ connected to each of the Voronoi nodes with an edge of length zero,

2. run any single source shortest path algorithm in the augmented graph $G^{\prime}$ with $v_{d}$ as its source, and

3. explore the search tree rooted at $v_{d}$ by following shortest path edges only.

This last step simulates a Dijkstra search by following the single source shortest path tree without using any expensive decrease-key operations (these operations have to be avoided to reduce the worst-case running time [Tho00b, Tho07]); a First-In-First-Out queue with constant time for the enqueue and dequeue operations is sufficient. For a pseudo-code description, see Algorithm 4. Although the construction is mainly of theoretical interest, it may be useful for example for parallel or distributed algorithms and for software that must rely on certain libraries.

Note that, if a single source shortest path algorithm $\mathcal{A}$ works for a special class of graphs $\mathcal{G}$, the augmented graph $G^{\prime}$ may not necessarily be in $\mathcal{G}$, and thus algorithm $\mathcal{A}$ cannot be used in general. For example, for planar graphs, the $O(n)$-time algorithm by Henzinger et al. [HKRS97] cannot be applied directly to compute the Voronoi diagram since planarity may be violated by adding a dummy node. In the particular case of the algorithm by Henzinger et al., however, the analysis of the running time depends on separators which do admit the introduction of a dummy node.

Theorem 1. Using any general single source shortest path algorithm with running time $t(n, m)$, Algorithm 4 computes a graph Voronoi dual in time $O(n+m+t(n, m))$.

Proof. After running the SSSP algorithm in time $t(n, m)$, Algorithm 4 visits every node exactly once and every edge exactly twice (once for each end point).

For undirected graphs we may use the $O(m)$-time SSSP algorithm by Thorup [Tho99, Tho00a]. 


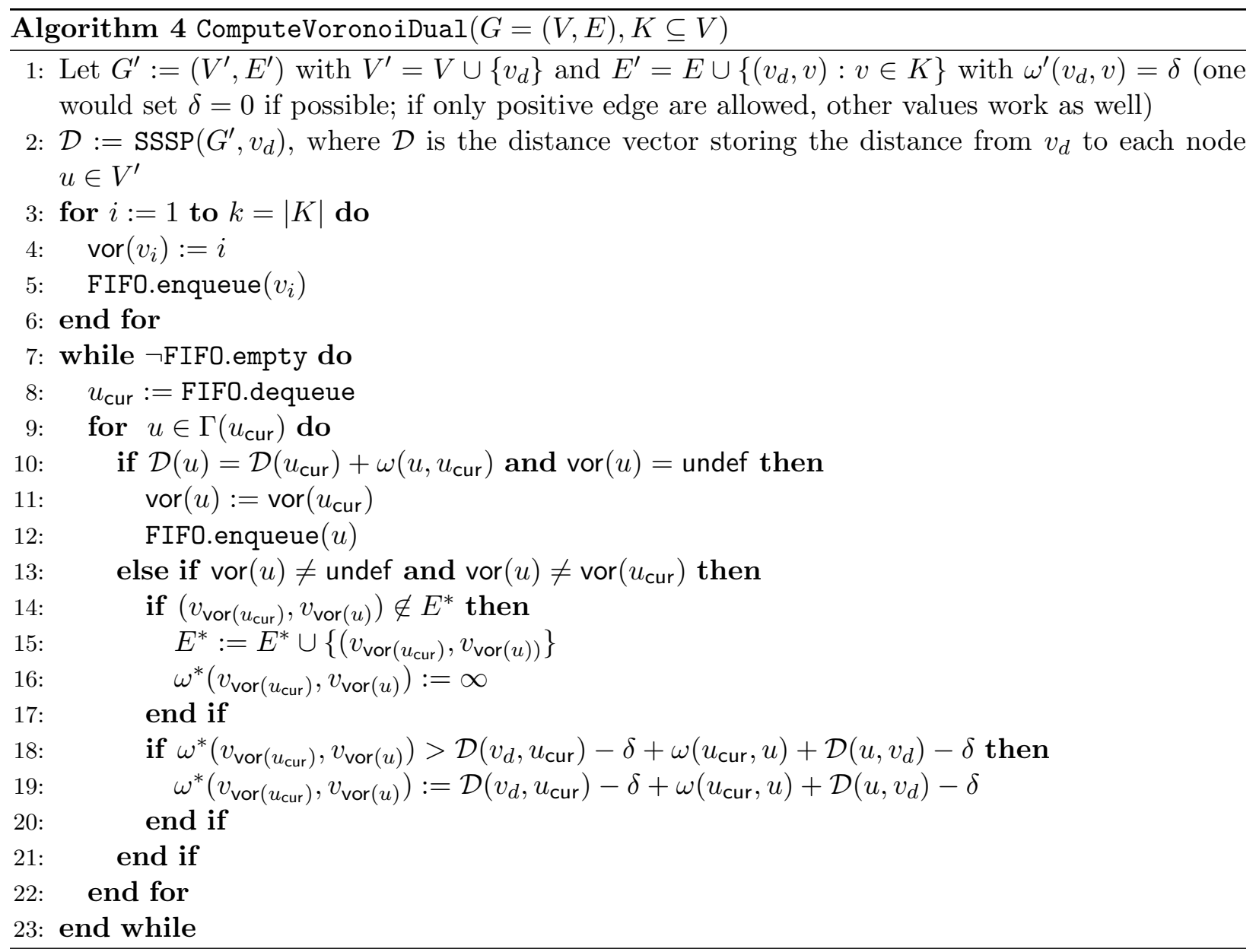

Corollary 2. For undirected graphs, the graph Voronoi dual can be computed in time $O(m+n)$ in the word RAM model.

Corollary 3. For a graph $G=(V, E)$ with $n:=|V|$ and $m:=|E|$, Algorithm 2 takes time proportional to that of Dijkstra's single source shortest path algorithm.

\section{$5 \quad$ Stretch Analysis}

In this section, we prove that the expected path length approximation ratio is logarithmic in the number of edges of an exact shortest path.

Theorem 2. For shortest paths having h edges, Algorithm 3, given a graph and its Voronoi dual with sampling rate $p$ (constructed by Algorithm 2), has expected approximation ratio $O\left(\lg _{1 /(1-p)} h\right)$.

The path $S P_{\mathcal{S}}(s, t)$ found by the algorithm is an approximation, since it is possible that no actual shortest path $S P_{G}(s, t)$ lies entirely within the Voronoi sleeve $\mathcal{S}$. We explain how this is possible, and give an upper bound on the expected length $\ell\left(S P_{\mathcal{S}}(s, t)\right)$. For this purpose, we prove relationships between the lengths of simple paths $P$ and their corresponding Voronoi paths $P^{*}$. The stretch of a path $P^{*}$ depends on the number and distribution of Voronoi nodes on the path 
$P$. In particular, the stretch depends linearly on the largest interval between two Voronoi nodes on the path.

Definition 5. For a path $P=\left(u_{0}, u_{1}, \ldots, u_{h}\right)$ in a graph $G=(V, E, \omega)$, and a set of Voronoi nodes $K \subseteq V$, two Voronoi nodes $v_{i}, v_{j}$ on $P$ are called consecutive if the subpath between $v_{i}$ and $v_{j}$ does not contain another Voronoi node. The gap $g$ between two consecutive Voronoi nodes on the path is defined as the number of edges of this subpath. The largest gap of a path is the maximum over all gaps between two consecutive Voronoi nodes on the path.

To simplify the analysis, we initially assume that $s$ and $t$ are Voronoi nodes. Later, we will relax this restriction.

We wish to prove that the stretch is at most the size of the largest gap $\bar{h}$ between two Voronoi nodes on the path $S P_{G}(s, t)$. For the analysis we fix a shortest path $S P_{G}(s, t)=\left(s, u_{1}, u_{2}, \ldots, u_{h-1}, t\right)$. If the corresponding Voronoi path $\left(S P_{G}(s, t)\right)^{*}$ is a shortest path from $s$ to $t$ in the Voronoi dual, then the Voronoi sleeve $\mathcal{S}$ also contains $S P_{G}(s, t)$. Figure 2 gives an example for which $\left(S P_{G}(s, t)\right)^{*}$ is not a shortest path in the dual.

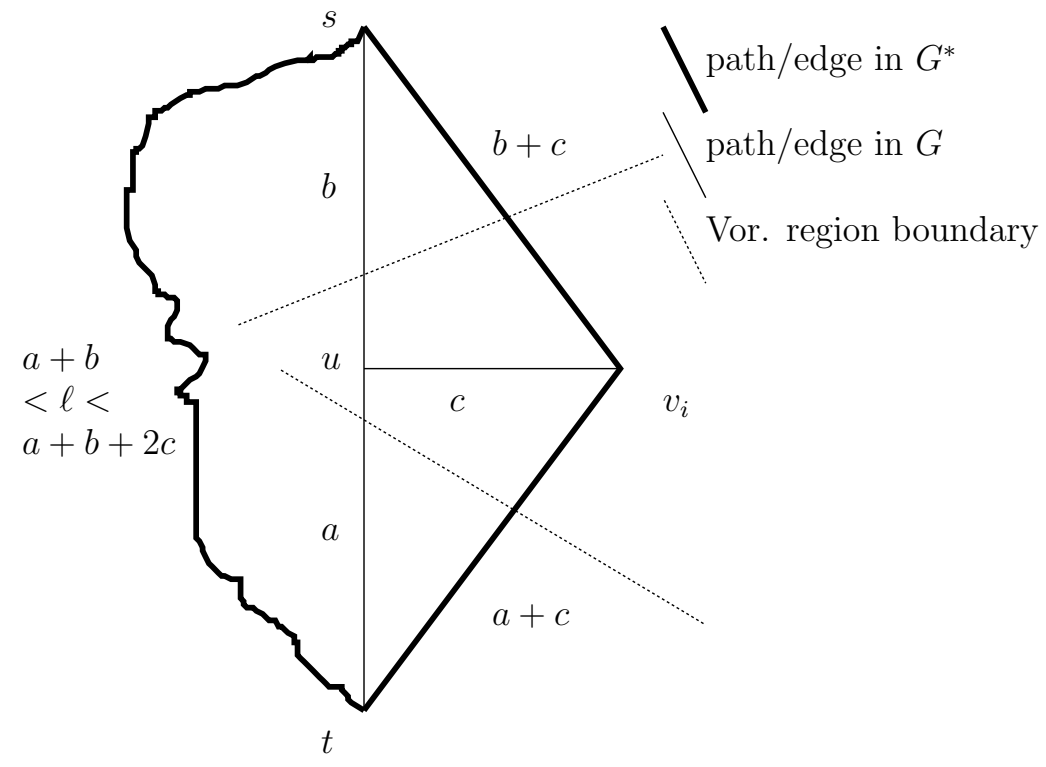

Figure 2: $s, t$, and $v_{i}$ are Voronoi nodes. The shortest path from $s$ to $t$ leads through $u$, which is in $v_{i}$ 's Voronoi region (if $c<a$ and $c<b$ ), and paths in the Voronoi dual pass through $v_{i}$. If $\ell<a+b+2 c$, the shortest path in the Voronoi dual $S P_{G^{*}}$ takes the left-hand route, and the Voronoi sleeve $\mathcal{S}$ does not contain $u$.

In Lemma 3, for any simple path $P$, we give a worst-case bound on the length of the corresponding Voronoi path. $P^{*}$ can have maximal stretch if there is no Voronoi node among the intermediate nodes and the corresponding Voronoi nodes have maximal distance (while still satisfying the Voronoi condition).

Lemma 3. Given a simple path $P=\left(s, u_{1}, \ldots, u_{h-1}, t\right)$ between two Voronoi nodes $s=u_{0}$ and $t=u_{h}$ with $h$ edges and length $\ell(P)$, the corresponding Voronoi path $P^{*}$ in the Voronoi dual $G^{*}$ has at most length $\ell\left(P^{*}\right) \leq h \cdot \ell(P)$. This upper bound is tight. 


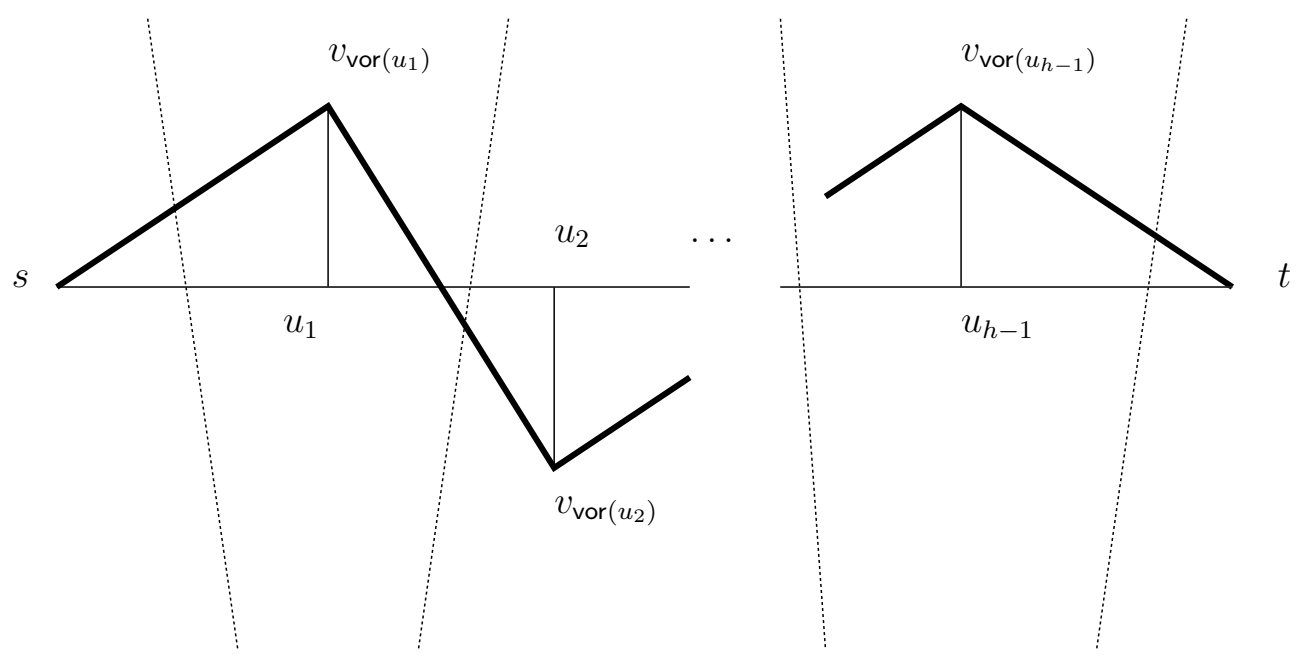

Figure 3: The shortest path between two Voronoi nodes $s$ and $t$ with $h-1$ intermediate nodes $u_{1}, \ldots, u_{h-1}$. The distance between two Voronoi nodes that are adjacent in the Voronoi dual is at $\operatorname{most} \omega^{*}\left(v_{\operatorname{vor}\left(u_{k}\right)}, v_{\operatorname{vor}\left(u_{k+1}\right)}\right) \leq d\left(v_{\operatorname{vor}\left(u_{k}\right)}, u_{k}\right)+\omega\left(u_{k}, u_{k+1}\right)+d\left(u_{k+1}, v_{\operatorname{vor}\left(u_{k+1}\right)}\right)$.

Proof. The path contains $h-1$ intermediate nodes and $h$ edges and therefore passes through at most $h+1$ different Voronoi regions. Out of these, at most $h-1$ regions are 'interfering' regions, meaning that the original shortest path does not lead through the corresponding Voronoi nodes but the shortest Voronoi path does. The path length $\ell(P)$ in the original graph is the sum of the edge weights $\ell(P):=d(s, t)=\sum_{k=0}^{h-1} \omega\left(u_{k}, u_{k+1}\right)$. The length $d^{*}\left(v_{\operatorname{vor}\left(u_{k}\right)}, v_{\operatorname{vor}\left(u_{k+1}\right)}\right)$ of an edge between two Voronoi nodes on the path $P^{*}$ can be bounded as follows (see Figure 3 ):

$$
d^{*}\left(v_{\operatorname{vor}\left(u_{k}\right)}, v_{\operatorname{vor}\left(u_{k+1}\right)}\right) \leq d\left(v_{\operatorname{vor}\left(u_{k}\right)}, u_{k}\right)+\omega\left(u_{k}, u_{k+1}\right)+d\left(u_{k+1}, v_{\operatorname{vor}\left(u_{k+1}\right)}\right)
$$

From the Voronoi condition, we observe that $\forall j: d\left(u_{k}, v_{\operatorname{vor}\left(u_{k}\right)}\right) \leq d\left(u_{k}, v_{\operatorname{vor}\left(u_{j}\right)}\right)$. Due to the assumption that $s$ and $t$ are also Voronoi nodes, this also holds for source and target. That is,

$$
\begin{aligned}
d\left(u_{k}, v_{\operatorname{vor}\left(u_{k}\right)}\right) & \leq d\left(s, u_{k}\right) \\
d\left(u_{k}, v_{\operatorname{vor}\left(u_{k}\right)}\right) & \leq d\left(u_{k}, t\right) \\
& =d\left(v_{\operatorname{vor}\left(u_{k}\right)}, u_{k}\right)
\end{aligned}
$$


This yields:

$$
\begin{aligned}
\ell\left(P^{*}\right) \leq d^{*}(s, t)= & d^{*}\left(s, v_{\operatorname{vor}\left(u_{1}\right)}\right) \\
& +\sum_{k=1}^{h-2}\left[d\left(v_{\operatorname{vor}\left(u_{k}\right)}, u_{k}\right)+\omega\left(u_{k}, u_{k+1}\right)+d\left(u_{k+1}, v_{\operatorname{vor}\left(u_{k+1}\right)}\right)\right] \\
& +d^{*}\left(v_{\operatorname{vor}\left(u_{h-1}\right)}, t\right) \\
\leq & \omega\left(s, u_{1}\right)+d\left(u_{1}, v_{\operatorname{vor}\left(u_{1}\right)}\right) \\
& +\sum_{k=1}^{h-2}\left[d\left(v_{\operatorname{vor}\left(u_{k}\right)}, u_{k}\right)+d\left(u_{k+1}, v_{\operatorname{vor}\left(u_{k+1}\right)}\right)\right] \\
& +\sum_{k=1}^{h-2} \omega\left(u_{k}, u_{k+1}\right) \\
& +d\left(v_{\operatorname{vor}\left(u_{h-1}\right)}, u_{h-1}\right)+\omega\left(u_{h-1}, t\right) \\
\leq & d(s, t)+\sum_{k=1}^{h-1}\left[d\left(s, u_{k}\right)+d\left(u_{k}, t\right)\right] \\
= & h \cdot \ell(P)
\end{aligned}
$$

There exist constructions for which the bound can be shown to be tight. For example, for any choice of $a>\epsilon>0$, the edge weights of $G$ may be chosen such that $d\left(u_{k}, v_{\operatorname{vor}\left(u_{k}\right)}\right)=a-\epsilon, \omega\left(u_{k}, u_{k+1}\right)=\epsilon$, and $\omega\left(s, u_{1}\right)=\omega\left(u_{h-1}, t\right)=a$. Path $P$ has length $2 a+(h-2) \epsilon$, and the Voronoi path $P^{*}$ has length $2 a+(h-2) \epsilon+2(h-1) \cdot(a-\epsilon)$. As $\epsilon \rightarrow 0$, the ratio $\ell\left(P^{*}\right) / \ell(P) \rightarrow h$.

If in addition to the endpoints there are Voronoi nodes on the shortest path, the maximum stretch is guaranteed to be smaller than the number of edges on the shortest path. In the following lemma, we prove that the maximum stretch is proportional to the largest gap between Voronoi nodes on the path. The proof is a simple composition of Lemma 3, and is supported by the illustration in Figure 3.

Lemma 4. Let $P=\left(v_{i}, u_{1}, \ldots, u_{h-1}, v_{j}\right)$ be a simple path of length $\ell(P)$ between two Voronoi nodes $v_{i}=u_{0}$ and $v_{j}=u_{h}$. Let $\bar{h}$ denote the largest gap of $P$. The corresponding Voronoi path $P^{*}$ in the Voronoi dual $G^{*}$ has at most length $\ell\left(P^{*}\right) \leq \bar{h} \cdot \ell(P)$. This upper bound is tight.

Proof. Suppose there are $2+\nu$ Voronoi nodes $u_{k}=v_{\operatorname{vor}\left(u_{k}\right)}$ on the path. The remaining $h-1-\nu$ nodes are non-Voronoi nodes. We cut the path $P$ into subpaths $P_{k}$ between Voronoi nodes. Let $h_{k}$ denote the number of edges between two consecutive Voronoi nodes, which is the number of edges of $P_{k}$. The Voronoi path is composed of $1+\nu$ segments $P_{k}$ between Voronoi nodes $\left(\sum_{k=0}^{\nu} \ell\left(P_{k}\right)=P\right.$, $\left.\sum_{k=0}^{\nu} h_{k}=h, \forall k: h_{k} \leq \bar{h}\right)$. Composition of Lemma 3 leads to the following bound on the path length:

$$
\sum_{k=0}^{\nu} h_{k} \ell\left(P_{k}\right) \leq \sum_{k=0}^{\nu} \max _{\kappa \in\{0, \ldots, \nu\}} h_{\kappa} \ell\left(P_{k}\right) \leq \bar{h} \cdot \ell(P) .
$$

Tightness can be shown with the same example as in the proof of Lemma 3.

Lemma 6 gives an upper bound on the expected size of the largest gap. We use the following lemma by Szpankowski and Rego [SR90] concerning the maximum of geometric random variables. 
Lemma 5 (Szpankowski and Rego [SR90, eq. (2.6) and (2.12)]). Let $X_{i}, i=1,2, \ldots, n$ be a set of i.i.d. random variables distributed according to the geometric distribution with parameter $p$. That $i s$, for every $i=1,2, \ldots, n$ and $k \in \mathbb{N}^{+}$,

$$
\begin{aligned}
\operatorname{Pr}\left[X_{i}=k\right] & =(1-p)^{k-1} p \\
\mathrm{E}\left[X_{i}\right] & =p^{-1} \\
\mathrm{E}\left[X_{i}^{2}\right] & =(2-p) p^{-2} .
\end{aligned}
$$

Let $M_{n}=\max \left\{X_{1}, X_{2}, \ldots, X_{n}\right\}$. The expected value of $M_{n}$ is

$$
\begin{aligned}
\mathrm{E}\left[M_{n}\right] & =-\sum_{k=1}^{n}(-1)^{k}\left(\begin{array}{l}
n \\
k
\end{array}\right) \frac{1}{1-(1-p)^{k}} \\
& =\lg _{1 /(1-p)} n+O(1) .
\end{aligned}
$$

Lemma 6. In a path of length $h-1$, where each node has been selected as a Voronoi node independently at random with probability $p$, the longest sequence of non-Voronoi nodes is of expected length at most $O\left(\lg _{1 /(1-p)} h\right)$.

Proof. The path can be seen as a sequence of coin tosses, for which we want to bound the expected length of the longest sequence of tails. This problem is known as the Longest Success-Run [EMK97, Ch. 8.5]. We wish to bound the expectation of the maximum of $N$ independent geometric random variables with probability $p$ and sum $h-1-N$ ( $N$ itself being a random variable).

To derive a bound on the expectation, we observe that by dropping the sum condition, and by taking the maximum over $h \geq N$ random variables, the maximum value obtained can only increase.

As of Lemma 5, the expectation of the maximum of $h$ geometric random variables with probability $p$ is known to be at most $O\left(\lg _{1 /(1-p)} h\right)$.

We now combine Lemmas 3, 4, and 6 to prove Theorem 2 .

Proof of Theorem 2. Consider first the case where $s$ and $t$ are both Voronoi nodes.

Let $\bar{h}$ denote the largest gap of some shortest path $S P_{G}(s, t)$. Lemma 4 implies that the corresponding Voronoi path $\left(S P_{G}(s, t)\right)^{*}$ has length at most $\bar{h} \cdot \ell\left(S P_{G}(s, t)\right)$. Trivially, the shortest path in the Voronoi dual is of length no more than that of the Voronoi path; that is, $\ell\left(\left(S P_{G}(s, t)\right)^{*}\right) \geq$ $\ell\left(S P_{G^{*}}(s, t)\right)$. The path $S P_{G^{*}}(s, t)$ in the Voronoi dual corresponds to a path $P^{\prime}$ of the same length in the Voronoi sleeve Sleeve $\left(S P_{G^{*}}(s, t)\right)$. Therefore,

$$
\begin{aligned}
\ell\left(S P_{\mathcal{S}}(s, t)\right) & \leq \ell\left(P^{\prime}\right) \\
& =\ell\left(S P_{G^{*}}(s, t)\right) \\
& \leq \ell\left(\left(S P_{G}(s, t)\right)^{*}\right) \\
& \leq \bar{h} \cdot \ell\left(S P_{G}(s, t)\right) .
\end{aligned}
$$

Recall that nodes are independently selected as Voronoi nodes with sampling rate $p$. For a shortest path with $h$ edges, the expected largest gap $\bar{h}$ is at most $O\left(\lg _{1 /(1-p)} h\right)$ by Lemma 6 .

For the case where either $s$ or $t$ (or both) are not Voronoi nodes, if the path returned by Algorithm 3 has been found in Step 1, it is optimal, and the result holds trivially. For the remainder of the proof we assume that the shortest path has not been found in Step 1. In this 
case, the path returned is at most as long as the shortest path $P_{\text {vor }}$ in $G$ from $s$ to $t$ having $S P_{\text {Sleeve }\left(S P_{G^{*}}\left(v_{\operatorname{vor}(s)}, v_{\operatorname{vor}(t)}\right)\right)}\left(v_{\operatorname{vor}(s)}, v_{\operatorname{vor}(t)}\right)$ as a subpath. In the following, we derive an upper bound on $\ell\left(P_{\text {vor }}\right)$ with respect to the number of edges on the shortest path between $s$ and $t$, denoted by $h^{\prime}$. We have that

$$
\ell\left(P_{\mathrm{vor}}\right) \leq d\left(s, v_{\operatorname{vor}(s)}\right)+d^{*}\left(v_{\operatorname{vor}(s)}, v_{\operatorname{vor}(t)}\right)+d\left(v_{\operatorname{vor}(t)}, t\right) .
$$

Since the shortest path from $s$ to $t$ has not already been found directly in Step 1, it must be true that both $d\left(s, v_{\operatorname{vor}(s)}\right) \leq d(s, t)$ and $d\left(s, v_{\operatorname{vor}(s)}\right) \leq d(s, t)$. It remains to bound the distance between $v_{\operatorname{vor}(s)}$ and $v_{\operatorname{vor}(t)}$ in the dual graph.

Observe that augmenting the graph $G$ with one edge $\left(u, v_{\operatorname{vor}(u)}\right)$ of weight $d\left(u, v_{\operatorname{vor}(u)}\right)$ for each non-Voronoi node $u \in V \backslash K$ affects neither the Voronoi diagram nor the Voronoi dual, since the nodes on the shortest path from $v_{\text {vor }(u)}$ to $u$ cannot be interfered with by another Voronoi node.

In the augmented primal graph, by the triangle inequality, we have that $d\left(v_{\operatorname{vor}(s)}, v_{\operatorname{vor}(t)}\right) \leq$ $d\left(v_{\operatorname{vor}(s)}, s\right)+d(s, t)+d\left(t, v_{\operatorname{vor}(t)}\right) \leq 3 d(s, t)$ using a path with at most $1+h^{\prime}+1$ edges. Therefore, the expected distance $d^{*}\left(v_{\operatorname{vor}(s)}, v_{\mathrm{vor}(t)}\right)$ is also bounded by $O\left(\lg h^{\prime}\right) \cdot 3 d(s, t)$. The bound for $P_{\text {vor }}$ follows directly.

This concludes the proof of Theorem 2 .

\section{Experiments}

In the following, we provide an experimental evaluation for our implementation of the Voronoi shortest path approximation method. The preprocessing and query times are compared with those of Dijkstra's algorithm and with those of related but exact methods.

\subsection{Algorithms}

\subsubsection{Benchmarking}

As the methods in our study were developed and compiled on different computers and architectures, a direct comparison with reported query times would not be meaningful. We measure the performance of the methods against the bidirectional version of Dijkstra's algorithm, in terms of the ratio of the number of nodes settled by Dijkstra's algorithm over the number of nodes settled by the Voronoi method. This ratio, which we will refer to as the speed-up of the method, can be used to evaluate the performance of Steps 1, 2, and 4 of Algorithm 3. In addition, we count the number of marked regions to account for Step 3.

The use of the Voronoi sleeve in Steps 3 and 4 of Algorithm 3 leads to practical improvements in accuracy; however, the example in Figure 2 shows that for general graphs the worst-case stretch does not improve. For all the experiments, we evaluate the method once using the refinement step and once with these Voronoi sleeve steps omitted. For the second type of queries, the reported distance is the sum of the distances from the query source to the Voronoi source, from the Voronoi source to the Voronoi target, and from the Voronoi target to the query target, as computed in Steps 1 and 2 of Algorithm 3.

\subsubsection{Voronoi method}

Our method using the Voronoi dual can be parameterized using the sampling probability $p$, the value of which determines the trade-off between approximation quality and speed-up. For the evaluation, 
we consider three values of the sampling probability $-p=1 / 2, p=n^{-1 / 2}$, and $p=n^{-2 / 3}-$ that produce Voronoi nodesets of expected sizes $n / 2, \sqrt{n}$, and $\sqrt[3]{n}$ respectively. The variants are referred to as VorHalf, VorRoot, and VorCubeRt.

\subsubsection{Other methods}

Sanders and Schultes [SS07a, Table 1] provide a detailed overview of methods for accelerated pointto-point shortest path queries in road networks. Bauer et al. $\left[\operatorname{BDS}^{+} 08\right.$, p. 13] list another set of methods and compare their performance on several transportation networks. We select some of the fastest methods for comparison with our algorithm. Unless stated otherwise, we will use the naming conventions of [SS07a, $\left.\mathrm{BDS}^{+} 08\right]$ to refer to these methods.

- Highway Hierarchies (HH) [SS06] are based on the observation that a certain class of edges (the 'highway' edges) tend to have greater representation among the portion of the shortest paths that are not in the vicinity of either the source or target. A recursive computation of these edges, paired with a contraction step, leads to a hierarchy of graphs that enables an impressive speed-up at query time. $\mathrm{HH}+$ dist denotes a variant of $\mathrm{HH}$ where all higher levels with at most $O(\sqrt{n})$ nodes are replaced by a single distance table. $\mathrm{HH}+\operatorname{dist}+\mathrm{A}^{*}$ is $\mathrm{HH}$ combined with $\mathrm{A}^{*}$ search and implemented with distance tables [DSSW06]. Highway Node Routing (HNR) [SS07b] is another variant of the Highway Hierarchies strategy.

- In the same spirit as HH, Transit Node Routing (TNR) $\left[\mathrm{BFM}^{+} 07\right]$ identifies a set of nodes (called 'transit' nodes) that often occur on shortest paths. A table storing the distances between all pairs of these nodes allows any shortest path distance to be computed with a small number of table look-ups. Two variants are listed: TNR-eco with economical space consumption, and TNR-gen with generous space consumption.

- The Arc-Flag method [Lau04] computes a partition of the graph and then, for each component and for each shortest path ending in that component, it labels the first edge. A variant of this method, SHARC [BD08], incorporates techniques developed for Highway Hierarchies.

- Contraction Hierarchies (CHHNR) [GSSD08] is an extension of highway hierarchies in which the graph is further simplified using contraction operations. Many variants have been proposed; we consider only the variant with the fastest preprocessing time, CHHNREDs1235, and the variant with the best speed-up, CHHNREvsQwL. The CHASE method [BDS ${ }^{+} 08$ ] integrates the Contraction Hierarchies and Arc-Flag methods.

- A method based on $\mathrm{A}^{*}$ search by Goldberg and Harrelson [GH05], which we will refer to as simply $\mathrm{A}^{*}$, is one of the first methods with reasonable preprocessing time and good speed-up.

- ALT-m16 [DW07] is a variant of ALT [GW05], which in turn is a combination of A*, Landmarks, and speed-up techniques based on the triangle inequality. CALT-m16 and CALTa64 $\left[\mathrm{BDS}^{+} 08\right]$ are two variants of a method that combines ALT and Contraction Hierarchies.

\subsection{Data sets}

For the sake of comparison, we consider transportation networks that were used by Sanders and Schultes [SS07a] and Bauer et al. $\left[\mathrm{BDS}^{+}\right.$08, BD08] in their evaluations. In addition, to demonstrate 
that our method is effective for more general graphs, we run experiments with a social network, a citation graph, a router network, and protein interaction networks as data sets. The node degrees of these graphs seem to follow a power-law distribution [Mit03].

\subsubsection{Road networks}

The road network of Western Europe has been made available for scientific use by the company PTV AG. It covers 14 countries and, with its massive size of 18,010,173 nodes and 42,560,279 directed edges, it serves as an important benchmark for shortest path queries. In order to apply the Voronoi method, we convert the graph into an undirected form. There are two different edge weightings, one representing geographical distances and the other representing driving time. We conduct experiments for both.

\subsubsection{Public transportation}

We also conduct experiments for three European public transportation networks: (1) long railway connections in Europe, with 1,586,862 nodes and 2,402,352 directed edges, (2) the bus network of the Rhein-Main-Verkehrsverbund RMV, with 2,278,066 nodes and 3,417,084 directed edges, and (3) the bus network of the Verkehrsverbund Berlin Brandenburg VBB, with 2,600,818 nodes and 3,901,212 directed edges. The graphs considered by [BDS $\left.{ }^{+} 08, \mathrm{BD} 08\right]$ differ slightly from those used for experimentation with the Voronoi method.

The numbers of nodes and edges of the RMV and VBB input graphs are nearly identical; however, the long railway graph used in our experimentation has $33 \%$ more nodes and edges than in $\left[\mathrm{BDS}^{+} 08, \mathrm{BD} 08\right]$. Again, for the Voronoi experimentation, the graphs were converted into an undirected form.

\subsubsection{Social networks}

We extracted the DBLP computer science bibliography [Ley02] co-author graph from an official XML version downloaded on 24 August 2008. In the graph, two authors are connected by an edge if they have at least one joint publication. This yielded an undirected graph, from which we selected the largest connected component. The final graph is unweighted and consists of 511,163 nodes and $1,871,070$ edges.

\subsubsection{Router topology}

CAIDA maintains data on the router-level topology of a portion of the Internet [Coo03]. After cleaning we obtained an undirected, unweighted graph with 190,914 nodes and 607,610 edges.

\subsubsection{Citation graph}

The citations for 27,400 publications in the high energy physics research literature were used as a data set in the KDD Cup 2003 competition [GSDF03]. From these citations, we constructed an undirected, unweighted graph with 352,542 edges. 


\subsubsection{Protein interactions}

The Database of Interacting Proteins $\left[\mathrm{SMS}^{+} 02\right]$ catalogs experimentally determined interactions among proteins. We extracted the largest connected component, consisting of 19,928 nodes and 82,406 edges. BioGRID is a general repository for interaction data sets $\left[\mathrm{SBR}^{+} 06\right]$ from which we extracted the largest connected component, consisting of 4,039 nodes and 43,854 edges.

\subsection{Experimental Setting}

In this section we describe the experimental setting for the Voronoi method. The implementation is written in $\mathrm{C}++$ and executed on one core of a $2 \times 2.66 \mathrm{GHz}$ Dual-Core Intel Xeon Desktop with 6 GB $800 \mathrm{MHz}$ DDR2 FB-DIMM running Mac OS X 10.5.6.

Every graph was preprocessed 1,000 times using different random seeds (250 times for the European road networks). For these runs we report the mean value and standard deviation of the execution time in seconds. After preprocessing, we performed 100 shortest path queries for random $(s, t)$ pairs. For these queries, we provide the mean values and standard deviations of the speed-up relative to the bidirectional version of Dijkstra's algorithm, and of the multiplicative stretch relative to a shortest path.

\subsection{Results}

Running times, speed-ups, and approximation qualities for the Voronoi method are listed in Table 2, for all data sets. The performances of the other methods are listed in Table 4 as originally summarized in [SS07a, GSSD08, $\mathrm{BDS}^{+} 08$ ].

Preprocessing For the Voronoi method, as Lemma 3 predicts, the preprocessing cost is extremely low for all three values of $p$. For the non-planar graphs, the greatest preprocessing times were observed for the largest value, $p=1 / 2$. This likely reflects the logarithmic cost of the heap operations associated with the computation of Voronoi regions. At the start of the Dijkstra search, the heap is initialized with all neighbors of the graph Voronoi nodes. When $p$ is large, the initial heap size is a large proportion of the total number of nodes, and the cost of the heap operations becomes significant. On the other hand, when $p$ and the average node degree are both small, the heap evolves smoothly with its size remaining small.

Speed-up For road networks VORHALF achieves moderate speed-ups of approximately 2, which likely reflects the fact that the expected number of nodes of the Voronoi dual is half that of the original graph. For the power-law graphs, probability $p=1 / 2$ does not lead to a significant speedup. One reason for this might be that the Voronoi dual for each of these graphs is quite dense and, as a consequence, the Dijkstra search in the dual explores many nodes until it can find the destination. For the smaller probabilities, larger speed-ups can be observed, but the performance gain is significantly smaller than the speed-ups obtained for almost planar networks. There, the speed-up seems proportional to $1 / p$. As expected, if for small values of $p$ the sleeve is used to refine the path, the speed-up decreases drastically due to the large size of this subgraph.

Stretch The Voronoi method achieved stretch values that were surprisingly consistent among different data sets, with most values under 2 and very close to optimal for the road networks. Figure 4 shows the approximate path length versus the shortest path length, with and without the sleeve refinement steps. The theoretical worst-case logarithmic dependency on the number of edges cannot be observed in the experimental results. Refinement using the sleeve substantially improves the stretch in practice, although the theoretical performance is not affected. 


\section{Conclusion}

We have presented a simple and general method based on Voronoi duals to efficiently support shortest path queries in undirected graphs with very low preprocessing overheads and competitive query times, at the cost of exactness. The method was shown to be effective on a variety of graph types while remaining a reasonable alternative to existing exact methods specifically designed for transportation networks. The results of our experiments also demonstrate that the approximation ratio in practice is significantly better than the tight theoretical worst-case bound proved in the main theorem of this paper. The maximal distortion of paths in the graph Voronoi dual depends on the distance between nodes in the original graph, unlike Delaunay triangulations of the Euclidean plane, which have constant distortion [DFS90, KG92].

An interesting topic for future research would be an expected-case analysis for weighted graphs from a variety of distributions.

It remains open as to whether the Voronoi method presented in this paper can be extended to handle directed graphs. The nature of the Voronoi dual within a directed graph is inherently different from the dual within an undirected graph. The need for path connectivity suggests the construction of two Voronoi diagrams, one where reachability paths are oriented outward from Voronoi nodes and another where reachability paths are oriented inward. As the respective Voronoi regions may not coincide [Erw00], it is not straightforward to define a single dual structure whose shortest path lengths approximate those of the original graph.

\section{References}

[ACS99] Karen Aardal, Fabian A. Chudak, and David B. Shmoys. A 3-approximation algorithm for the $k$-level uncapacitated facility location problem. Information Processing Letters, 72:161-167, 1999.

[AGK $\left.{ }^{+} 04\right]$ Vijay Arya, Naveen Garg, Rohit Khandekar, Adam Meyerson, Kamesh Munagala, and Vinayaka Pandit. Local search heuristics for $k$-median and facility location problems. SIAM Journal on Computing, 33(3):544-562, 2004. Announced at STOC 2001.

[Aur91] Franz Aurenhammer. Voronoi diagrams - a survey of a fundamental geometric data structure. ACM Computing Surveys, 23(3):345-405, 1991.

[BD08] Reinhard Bauer and Daniel Delling. SHARC: Fast and robust unidirectional routing. In Proceedings of the 10th Workshop on Algorithm Engineering and Experiments (ALENEX'08), pages 13-26, 2008.

$\left[\mathrm{BDS}^{+} 08\right]$ Reinhard Bauer, Daniel Delling, Peter Sanders, Dennis Schieferdecker, Dominik Schultes, and Dorothea Wagner. Combining hierarchical and goal-directed speed-up techniques for Dijkstra's algorithm. In Experimental Algorithms, 7th International Workshop (WEA'08), Provincetown, MA, USA, May 30-June 1, 2008, Proceedings, pages $303-318,2008$.

$\left[\mathrm{BFM}^{+} 07\right]$ Holger Bast, Stefan Funke, Domagoj Matijevic, Peter Sanders, and Dominik Schultes. In transit to constant time shortest-path queries in road networks. In Proceedings of the Workshop on Algorithm Engineering and Experiments (ALENEX'07), New Orleans, Louisiana, USA, January 6, 2007, 2007. 
[BGSU08] Surender Baswana, Akshay Gaur, Sandeep Sen, and Jayant Upadhyay. Distance oracles for unweighted graphs: Breaking the quadratic barrier with constant additive error. In Automata, Languages and Programming, 35th International Colloquium, ICALP 2008, Reykjavik, Iceland, July 7-11, 2008, Proceedings, Part I: Tack A: Algorithms, Automata, Complexity, and Games, pages 609-621, 2008.

[BK06] Surender Baswana and Telikepalli Kavitha. Faster algorithms for approximate distance oracles and all-pairs small stretch paths. In 47th Annual IEEE Symposium on Foundations of Computer Science (FOCS 2006), 21-24 October 2006, Berkeley, California, USA, pages 591-602, 2006.

[Cha07] Timothy M. Chan. More algorithms for all-pairs shortest paths in weighted graphs. In Proceedings of the 39th Annual ACM Symposium on Theory of Computing (STOC'07), pages 590-598, 2007.

[Coo03] Cooperative Association for Internet Data Analysis. Router-level topology measurements. Online at http://www.caida.org/tools/measurement/skitter/router_topology/, file: itdk0304_rlinks_undirected.gz, 2003.

[CP07] Timothy M. Chan and Mihai Patrascu. Voronoi diagrams in $n \cdot 2^{O(\sqrt{\lg \lg n})} \operatorname{time}$. In Proceedings of the 39th Annual ACM Symposium on Theory of Computing, San Diego, California, USA, June 11-13, 200\%, pages 31-39, 2007.

[DFS90] David P. Dobkin, Steven J. Friedman, and Kenneth J. Supowit. Delaunay graphs are almost as good as complete graphs. Discrete $\mathcal{E}$ Computational Geometry, 5:399-407, 1990 .

[Dij59] Edsger Wybe Dijkstra. A note on two problems in connexion with graphs. Numerische Mathematik, 1:269-271, 1959.

[Dir50] Gustav Lejeune Dirichlet. Über die Reduktion der positiven quadratischen Formen mit drei unbestimmten ganzen Zahlen. Journal für die Reine und Angewandte Mathematik, 40:209-227, 1850.

[Dor67] Jim E. Doran. An approach to automatic problem-solving. Machine Intelligence, 1:105$124,1967$.

[DSSW06] Daniel Delling, Peter Sanders, Dominik Schultes, and Dorothea Wagner. Highway hierarchies star. In 9th DIMACS Implementation Challenge, 2006.

[DW07] Daniel Delling and Dorothea Wagner. Landmark-based routing in dynamic graphs. In Experimental Algorithms, 6th International Workshop (WEA'07), Rome, Italy, June 6-8, 2007, Proceedings, pages 52-65, 2007.

[EG08] David Eppstein and Michael T. Goodrich. Studying (non-planar) road networks through an algorithmic lens. In 16th ACM SIGSPATIAL International Symposium on Advances in Geographic Information Systems, ACM-GIS 2008, November 5-7, 2008, Irvine, California, USA, Proceedings, page 16, 2008. 
[EMK97] Paul Embrechts, Thomas Mikosch, and Claudia Klüppelberg. Modelling extremal events: for insurance and finance. Springer-Verlag, London, UK, 1997.

[EP04] Michael Elkin and David Peleg. $(1+\epsilon, \beta)$-spanner constructions for general graphs. SIAM Journal on Computing, 33(3):608-631, 2004. Announced at STOC 2001.

[Erw00] Martin Erwig. The graph Voronoi diagram with applications. Networks, 36(3):156-163, 2000 .

[FT87] Michael L. Fredman and Robert Endre Tarjan. Fibonacci heaps and their uses in improved network optimization algorithms. Journal of the ACM, 34(3):596-615, 1987. Announced at FOCS 1984.

[FW93] Michael L. Fredman and Dan E. Willard. Surpassing the information theoretic bound with fusion trees. Journal of Computer and System Sciences, 47(3):424-436, 1993.

[GH05] Andrew V. Goldberg and Chris Harrelson. Computing the shortest path: A* search meets graph theory. In Proceedings of the Sixteenth Annual ACM-SIAM Symposium on Discrete Algorithms (SODA'05), Vancouver, British Columbia, Canada, January 23-25, 2005, pages 156-165, 2005.

[GKP05] Naveen Garg, Rohit Khandekar, and Vinayaka Pandit. Improved approximation for universal facility location. In Proceedings of the Sixteenth Annual ACM-SIAM Symposium on Discrete Algorithms, (SODA'05), Vancouver, British Columbia, Canada, January 23-25, 2005, pages 959-960, 2005.

[GSDF03] Lise Getoor, Ted E. Senator, Pedro Domingos, and Christos Faloutsos, editors. SIGKDD Proceedings, 2003.

[GSSD08] Robert Geisberger, Peter Sanders, Dominik Schultes, and Daniel Delling. Contraction hierarchies: Faster and simpler hierarchical routing in road networks. In Experimental Algorithms, 7th International Workshop (WEA'08), Provincetown, MA, USA, May 30June 1, 2008, Proceedings, pages 319-333, 2008.

[GW05] Andrew V. Goldberg and Renato Fonseca F. Werneck. Computing point-to-point shortest paths from external memory. In Proceedings of the Seventh Workshop on Algorithm Engineering and Experiments (ALENEX'05), pages 26-40, 2005.

[HKRS97] Monika Rauch Henzinger, Philip N. Klein, Satish Rao, and Sairam Subramanian. Faster shortest-path algorithms for planar graphs. Journal of Computer and System Sciences, 55(1):3-23, 1997. Announced at STOC 1994.

[KG92] J. Mark Keil and Carl A. Gutwin. Classes of graphs which approximate the complete Euclidean graph. Discrete \& Computational Geometry, 7:13-28, 1992.

[Lau04] Ulrich Lauther. An extremely fast, exact algorithm for finding shortest paths in static networks with geographical background. In Geoinformation und Mobilität - von der Forschung zur praktischen Anwendung, volume 22, pages 219-230, 2004. 
[Ley02] Michael Ley. The DBLP computer science bibliography: Evolution, research issues, perspectives. In String Processing and Information Retrieval, 9th International Symposium (SPIRE'02), Lisbon, Portugal, September 11-13, 2002, Proceedings, pages 1-10, 2002 .

[Meh88] Kurt Mehlhorn. A faster approximation algorithm for the Steiner problem in graphs. Information Processing Letters, 27(3):125-128, 1988.

[Mit03] Michael Mitzenmacher. A brief history of generative models for power law and lognormal distributions. Internet Mathematics, 1(2), 2003.

[Ram96] Rajeev Raman. Priority queues: Small, monotone and trans-dichotomous. In Algorithms - ESA '96, Fourth Annual European Symposium, Barcelona, Spain, September 25-27, 1996, Proceedings, pages 121-137, 1996.

[Ram97] Rajeev Raman. Recent results on the single-source shortest paths problem. SIGACT News, 28:81-87, 1997.

[SBR ${ }^{+}$06] Chris Stark, Bobby-Joe Breitkreutz, Teresa Reguly, Lorrie Boucher, Ashton Breitkreutz, and Mike Tyers. Biogrid: a general repository for interaction datasets. Nucleic Acids Research, 34(1):535-539, 2006.

[Sha75] Michael Ian Shamos. Geometric complexity. In Conference Record of Seventh Annual ACM Symposium on Theory of Computation (STOC'75), 5-7 May 1975, Albuquerque, New Mexico, USA, pages 224-233, 1975.

$\left[\mathrm{SMS}^{+}\right.$02] Lukasz Salwinski, Christopher S. Miller, Adam J. Smith, Frank K. Pettit, James U. Bowie, and David Eisenberg. DIP, the database of interacting proteins: a research tool for studying cellular networks of protein interactions. Nucleic Acids Research, 30(1):303-305, 2002.

[SR90] Wojciech Szpankowski and Vernon Rego. Yet another application of a binomial recurrence. Order statistics. Computing, 43(4):401-410, 1990.

[SS06] Peter Sanders and Dominik Schultes. Engineering highway hierarchies. In Algorithms - ESA 2006, 14th Annual European Symposium, Zurich, Switzerland, September 11-13, 2006, Proceedings, pages 804-816, 2006.

[SS07a] Peter Sanders and Dominik Schultes. Engineering fast route planning algorithms. In Experimental Algorithms, 6th International Workshop (WEA'07), Rome, Italy, June 6-8, 2007, Proceedings, pages 23-36, 2007.

[SS07b] Dominik Schultes and Peter Sanders. Dynamic highway-node routing. In Experimental Algorithms, 6th International Workshop (WEA'07), Rome, Italy, June 6-8, 2007, Proceedings, pages 66-79, 2007.

[Svi08] Zoya Svitkina. Lower-bounded facility location. In Proceedings of the Nineteenth Annual ACM-SIAM Symposium on Discrete Algorithms (SODA'08), San Francisco, California, USA, January 20-22, 2008, pages 1154-1163, 2008. 
[Tho99] Mikkel Thorup. Undirected single-source shortest paths with positive integer weights in linear time. Journal of the ACM, 46(3):362-394, 1999. Announced at FOCS 1997.

[Tho00a] Mikkel Thorup. Floats, integers, and single source shortest paths. Journal of Algorithms, 35(2):189-201, 2000. Announced at STACS 1998.

[Tho00b] Mikkel Thorup. On RAM priority queues. SIAM Journal of Computing, 30(1):86-109, 2000. Announced at SODA 1996.

[Tho04a] Mikkel Thorup. Compact oracles for reachability and approximate distances in planar digraphs. Journal of the ACM, 51(6):993-1024, 2004. Announced at FOCS 2001.

[Tho04b] Mikkel Thorup. Integer priority queues with decrease key in constant time and the single source shortest paths problem. Journal of Computer and System Sciences, 69(3):330353, 2004. Announced at STOC 2003.

[Tho07] Mikkel Thorup. Equivalence between priority queues and sorting. Journal of the ACM, 54(6), 2007. Announced at FOCS 2002.

[TZ05] Mikkel Thorup and Uri Zwick. Approximate distance oracles. Journal of the ACM, 52(1):1-24, 2005. Announced at STOC 2001.

[Vor07] Georgy Voronoi. Nouvelles applications des paramètres continus à la théorie des formes quadratiques. Journal für die Reine und Angewandte Mathematik, 133:97-178, 1907.

[Wil64] J. W. J. Williams. Algorithm 232: Heapsort. Communications of the ACM, 7:347-348, 1964. 


\begin{tabular}{|c|c|c|c|c|c|}
\hline \multirow[t]{2}{*}{ method } & \multirow[t]{2}{*}{ preprocessing $[s]$} & \multicolumn{2}{|c|}{ without sleeve } & \multicolumn{2}{|c|}{ with sleeve } \\
\hline & & speed-up & stretch & speed-up & stretch \\
\hline \multicolumn{6}{|c|}{ PTV European road network, driving time, $18,010,173$ nodes, $42,560,279$ edges } \\
\hline$\overline{\text { VORHALF }}$ & $31.7686 \pm 4.4436$ & $2.6061 \pm$ & $1.0394 \pm 0.0131$ & $2.5878 \pm 0.0750$ & $1.0111 \pm 0.0062$ \\
\hline VORROOT & $40.5296 \pm 3.6423$ & $3,518.0645 \pm$ & $1.6613 \pm 0.2078$ & $4.9991 \pm 4.9017$ & $1.1291 \pm 0.0783$ \\
\hline VORCuBeRT & $31.3372 \pm 2.8181$ & $39,918.4988 \pm 14,207.5395$ & $1.5544 \pm 0.4292$ & $1.5863 \pm 1.1123$ & $1.0405 \pm 0.0597$ \\
\hline \multicolumn{6}{|c|}{ PTV European road network, geographical distance, $18,010,173$ nodes, $42,560,279$ edges } \\
\hline$\overline{\text { VORHALF }}$ & 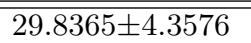 & 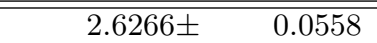 & 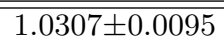 & 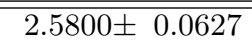 & $1.0139 \pm 0.0057$ \\
\hline VORROOT & $34.2785 \pm 3.0609$ & $3,672.4070 \pm$ & $1.1821 \pm 0.0960$ & $5.9212 \pm 7.9921$ & $1.0390 \pm 0.0249$ \\
\hline VORCUBeRt & $22.5531 \pm 2.0284$ & $42,266.6442 \pm 13,530.5983$ & $1.2882 \pm 0.5384$ & $1.6383 \pm 1.4232$ & $1.0141 \pm 0.0291$ \\
\hline \multicolumn{6}{|c|}{ Public transportation, long distance railway, $1,586,862$ nodes, $2,402,352$ edges } \\
\hline VORHALF & $2.0499 \pm 0.1998$ & $1.9511 \pm$ & $1.0180 \pm 0.0227$ & $1.8972 \pm 0.1367$ & $1.0080 \pm 0.0143$ \\
\hline VORROOT & $1.9086 \pm 0.0946$ & $363.8390 \pm$ & $1.3813 \pm 0.2848$ & $2.8527 \pm 3.3113$ & $1.0829 \pm 0.0971$ \\
\hline VORCUBeRT & $1.7633 \pm 0.0860$ & $2,116.0373 \pm 1,251.1773$ & $1.5167 \pm 0.6610$ & $1.2599 \pm 0.5990$ & $1.0247 \pm 0.0658$ \\
\hline \multicolumn{6}{|c|}{ Public transportation, RMV, 2,278,066 nodes, $3,417,084$ edges } \\
\hline VORHALF & $3.7714 \pm 0.4064$ & 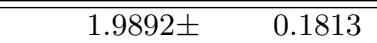 & $1.0290 \pm 0.0255$ & 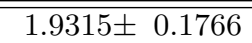 & $1.0104 \pm 0.0131$ \\
\hline Vor & $3.7455 \pm 0.2158$ & $789.2912 \pm$ & $1.2972 \pm 0.2591$ & $3.1802 \pm 5.4237$ & $=0.0864$ \\
\hline VORCuBeRt & $3.4120 \pm 0.1633$ & $5,973.7950 \pm 3,748.1389$ & $1.3522 \pm 0.6003$ & $1.3089 \pm 0.9703$ & $1.0204 \pm 0.0583$ \\
\hline \multicolumn{6}{|c|}{ Public transportation, VBB, 2,600,818 nodes, 3,901,212 edges } \\
\hline VORHALF & $4.1409 \pm 0.4180$ & $1.9881 \pm$ & $1.0335 \pm 0.0248$ & $1.9313 \pm 0.5172$ & $1.0075 \pm 0.0097$ \\
\hline & $2 \pm 0.2914$ & $866.8917 \pm \quad 405.4821$ & $1.4042 \pm 0.2516$ & $3.7864 \pm 7.6010$ & \pm 0.1000 \\
\hline VorCubeRt & $3.7145 \pm 0.2333$ & $7,373.2971 \pm 4,742.2783$ & $1.4375 \pm 3.3690$ & $1.3427 \pm 1.2759$ & $1.0244 \pm 0.0660$ \\
\hline \multicolumn{6}{|c|}{$\begin{array}{c}\text { DBLP co-authorship, 511,163 nodes, 1,871,070 edges } \\
\end{array}$} \\
\hline VORHALF & $0.9145 \pm 0.0431$ & $\begin{array}{ll}1.3576 \pm & 1.4690\end{array}$ & $1.2093 \pm 0.1805$ & $1.3447 \pm 1.4364$ & $1.1419 \pm 0.1468$ \\
\hline VORROOT & $0.8376 \pm 0.0430$ & $37.7082 \pm$ & $1.9323 \pm 0.3591$ & $11.4432 \pm 14.8387$ & $1.3954 \pm 0.2850$ \\
\hline VorCubeRt & $0.6041 \pm 0.0312$ & $143.8757 \pm$ & $2.0033 \pm 0.3630$ & $9.9616 \pm 12.5412$ & $1.2881 \pm 0.2406$ \\
\hline \multicolumn{6}{|c|}{ CAIDA router topology, 190,914 nodes, 607,610 edges } \\
\hline VORHALF & 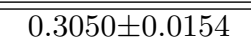 & $\begin{array}{ccc}1.3164 \pm & 1.1720\end{array}$ & $\begin{array}{l}1.1810 \pm 0.1703 \\
\end{array}$ & " & $1.1283 \pm 0.1359$ \\
\hline VORROOT & $0.1793 \pm 0.0092$ & $42.4832 \pm$ & $1.7845 \pm 0.3533$ & $7.8865 \pm 8.8062$ & $1.2345 \pm 0.2175$ \\
\hline VORCUBERT & $0.1562 \pm 0.0081$ & $135.5521 \pm$ & $1.8314 \pm 0.3755$ & $6.0451 \pm 7.1000$ & $1.1621 \pm 0.1837$ \\
\hline \multicolumn{6}{|c|}{ High energy physics citations, 27,400 nodes, 352,542 edges } \\
\hline$\overline{\text { VORHALF }}$ & 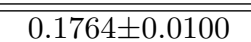 & $\begin{array}{ccc}1.6620 \pm & 1.2240\end{array}$ & $\begin{array}{l}1.3179 \pm 0.2909 \\
\end{array}$ & 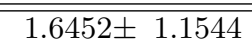 & $\bar{~} 1.2107 \pm 0.2323$ \\
\hline VORROOT & $0.0611 \pm 0.0043$ & $40.1114 \pm$ & $1.9918 \pm 0.4695$ & $11.5248 \pm 7.9582$ & $1.3390 \pm 0.3286$ \\
\hline VorCubeRt & $0.0461 \pm 0.0032$ & $101.9210 \pm$ & $2.0330 \pm 0.4852$ & $9.0423 \pm 7.5795$ & $1.2325 \pm 0.2750$ \\
\hline \multicolumn{6}{|c|}{ Database of Interacting Proteins, 19,928 nodes, 82,406 edges } \\
\hline VORHALF & $0.0117 \pm 0.0007$ & $2.2044 \pm$ & $1.1887 \pm 0.2188$ & $2.1248 \pm 1.0093$ & $1.1183 \pm 0.1778$ \\
\hline VORROOT & $0.0108 \pm 0.0007$ & $57.7343 \pm$ & $1.8214 \pm 0.4084$ & $9.1154 \pm 6.0720$ & $1.3216 \pm 0.3030$ \\
\hline VORCubeRT & $0.0096 \pm 0.0006$ & $134.4816 \pm$ & $1.9277 \pm 0.4444$ & $6.2541 \pm 3.8117$ & $1.2644 \pm 0.2703$ \\
\hline \multicolumn{6}{|c|}{ BioGRID, 4,039 nodes, 43,854 edges } \\
\hline VORHALF & $0.0035 \pm 0.0002$ & $1.5086 \pm$ & $1.2581 \pm 0.2718$ & $1.3722 \pm 0.6858$ & $1.1334 \pm 0.1973$ \\
\hline & $0.0025 \pm 0.0001$ & $10.7295 \pm$ & $1.8676 \pm 0.5737$ & $3.0394 \pm 1.9172$ & $1.2753 \pm 0.3354$ \\
\hline VORCuBeRt & $0.0024 \pm 0.0001$ & $18.6805 \pm$ & $1.9412 \pm 0.6250$ & $2.7906 \pm 1.7177$ & $1.2308 \pm 0.3137$ \\
\hline
\end{tabular}

Table 2: Experimental results for the Voronoi method. 

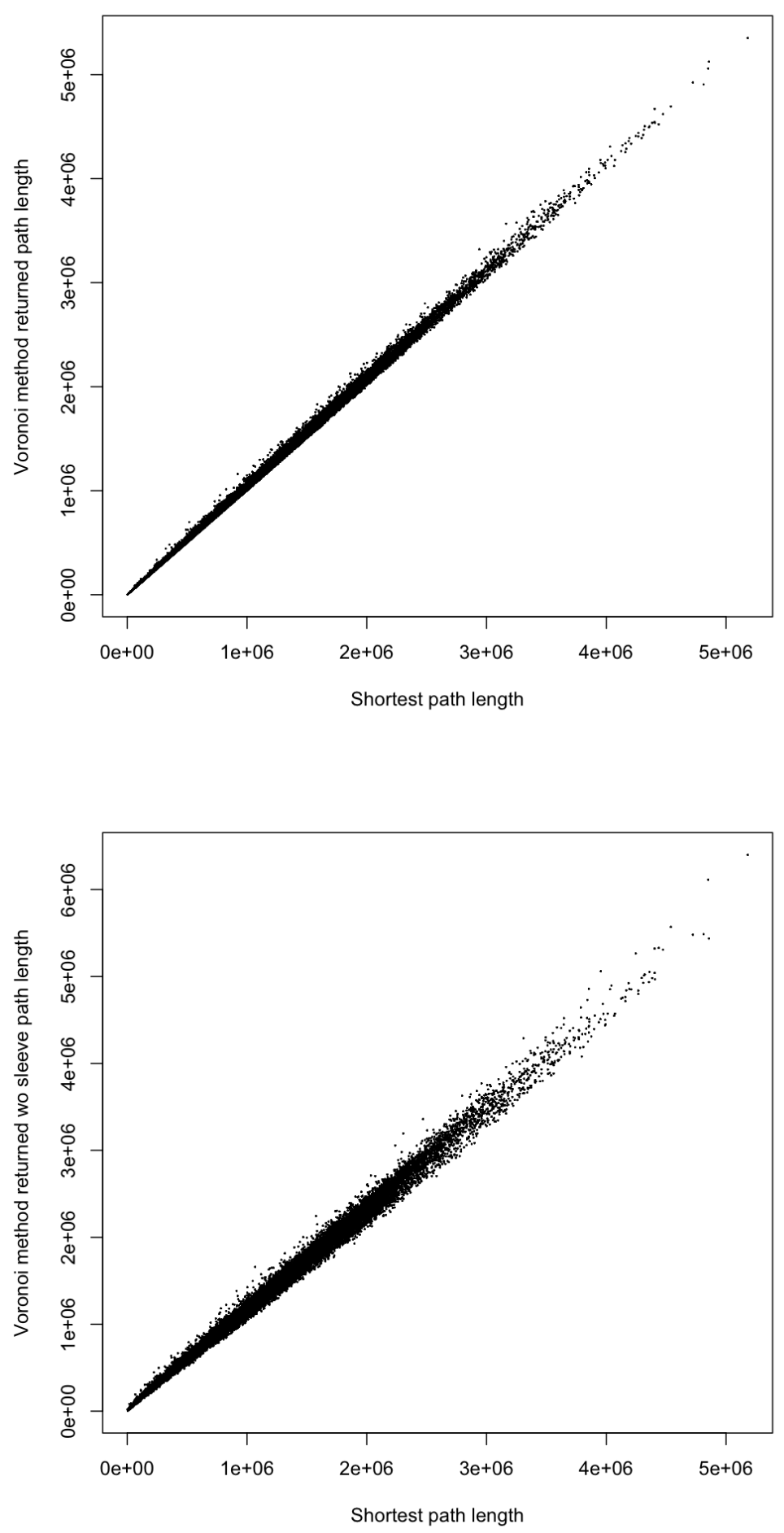

Figure 4: Approximate path length versus actual shortest path length for VorRoot on the European road network, distance metric. Top: using sleeve. Bottom: with sleeve steps omitted. The theoretical worst-case logarithmic dependency on the number of edges cannot be observed in the experimental results. Refinement using the sleeve substantially improves the stretch in practice, although the theoretical performance is not affected. 


\begin{tabular}{|lr||r|r|}
\hline \multicolumn{4}{|c|}{ PTV European road network, driving time } \\
\hline & & prep. $[s]$ & speed-up \\
\hline \hline CHHNREDS1235 & [GSSD08] & 602 & $\approx 8,505$ \\
A $^{*}$ & {$[$ GH05] } & 780 & 28 \\
HH & {$[$ SS06] } & 780 & 4,002 \\
HH+dist & [SS06] & 900 & 8,320 \\
HH+dist+A & [DSSW06] & 1,320 & 11,496 \\
HNR & [SSO7b] & 1,440 & 4,079 \\
CHHNREvSQwL & [GSSD08] & 1,914 & $\approx 10,874$ \\
TNR-eco & {$\left[\mathrm{BFM}^{+} 07\right]$} & 2,760 & 471,881 \\
TNR-gen & {$\left[\mathrm{BFM}^{+} 07\right]$} & 9,840 & $1,129,143$ \\
\hline
\end{tabular}

Table 3: Road networks: This table is excerpted from Sanders and Schultes [SS07a, Table 1] except for CHHNR values, which are from [GSSD08, Table 1]. Preprocessing times are converted from minutes to seconds to ease comparison with our method. Machines used (except for A*): 2.0 or $2.6 \mathrm{GHz}$ processor, 8 or $16 \mathrm{~GB}$ RAM, C++ implementation.

\begin{tabular}{|c|c|c|c|c|c|c|c|}
\hline $\begin{array}{l}|V| \\
|E|\end{array}$ & & \multicolumn{2}{|c|}{$\begin{array}{r}\text { long distance rail } \\
1,192,736 \\
1,789,088 \\
\end{array}$} & & $\begin{array}{l}\mathrm{MV} \\
2,277,812 \\
3,416,552\end{array}$ & & $\begin{array}{l}\mathrm{B} \\
2,599,953 \\
3,899,807\end{array}$ \\
\hline & & prep. $[s]$ & speed-up & prep. $[s]$ & speed-up & prep. $[s]$ & speed-up \\
\hline CALT-a64 & {$\left[\mathrm{BDS}^{+} 08\right]$} & 87 & 291.84 & 191 & 267.11 & 123 & 459.30 \\
\hline CALT-m16 & {$\left[\mathrm{BDS}^{+} 08\right]$} & 158 & 182.71 & 377 & 159.62 & 174 & 281.23 \\
\hline ALT-m16 & [DW07] & 291 & 20.30 & 556 & 18.91 & 604 & 23.04 \\
\hline CHHNR & [GSSD08] & 286 & $1,620.62$ & 2,584 & $2,077.69$ & 1,636 & $3,124.59$ \\
\hline CHASE & {$\left[\mathrm{BDS}^{+} 08\right]$} & 536 & $2,660.93$ & 2,863 & $4,649.26$ & 2,008 & $10,398.64$ \\
\hline SHARC & [BD08] & 12,540 & 81.04 & & & 36,120 & 118.10 \\
\hline
\end{tabular}

Table 4: Public transportation networks: This table is excerpted from Bauer et al. $\left[\mathrm{BDS}^{+} 08\right.$, p. 13]. SHARC is evaluated in [BD08, p. 10]. The speed-up is computed according to the number of settled nodes. Machines used: 2.0 or $2.6 \mathrm{GHz}$ processor, 8 or $16 \mathrm{~GB}$ RAM, C++ implementation. 